\title{
Parametric and Model Uncertainties Induced by Reduced Order Chemical Mechanisms for Biogas Combustion
}

\author{
Rodolfo S. M. Freitas ${ }^{a}$, Fernando A. Rochinha ${ }^{a}$, Daniel Mira ${ }^{b}$ and Xi Jiang ${ }^{c, *}$
}

${ }^{a}$ COPPE, Federal University of Rio de Janeiro, Rio de Janeiro, Rio de Janeiro, 21941-598, Brazil

${ }^{b}$ Barcelona Supercomputing Center (BSC), Barcelona, Spain

${ }^{c}$ School of Engineering \& Materials Science, Queen Mary University of London, Mile End Road, London E1 4NS, UK

\section{ARTICLE INFO}

\section{Keywords:}

Biogas Combustion

Machine learning

Model error

Surrogate modeling

Uncertainty quantification

\begin{abstract}
A B S TR A C T
This study investigates the impact of chemical kinetic uncertainties on biogas combustion using a Uncertainty Quantification (UQ)-based methodology. The results indicate that the variation of physicochemical properties introduced by composition variability introduces smaller uncertainties in the resulting flame properties than the Arrhenius parameters involved in the kinetics used to describe the oxidation process. We demonstrate that the use of reduced mechanisms for methane-air oxidation could be a starting point to develop optimized schemes for biogas combustion. In that regard, we adopted an embedded discrepancy approach to understanding the limits of the use of a reduced mechanism for methane/air in this renewable fuel. This strategy provides a way to reduce systematically the cost of reaction kinetics in simulations, while quantifying the accuracy of predictions of important target quantities. Finally, we develop a surrogate model for biogas flame propagation using machine learning techniques to make feasible a broader UQ analysis.
\end{abstract}

\section{Introduction}

In power and propulsion applications such as engines, the thermal energy contained in the fuel is usually converted to heat through combustion. This process is responsible not only for determining the rates of heat release and power output but also for dictating the formation of pollutants. In practical applications, there is a complex interaction between the chemical reactions and the flow field, especially when the flow is turbulent. There are many studies dedicated to developing computational predictive models for turbulent reacting flows [1,2], with the latter showing a high influence of the flow by the chemistry. The application of Computational Fluid Dynamics (CFD) to combustion science has been impeded by the complexity of chemistry modeling. The development of reliable and efficient chemical kinetic models is of primary relevance for the design and optimization of combustion systems. While CFD has been traditionally used in the context of Reynolds-averaged Navier-Stokes (RANS), the application of large-eddy-simulation (LES) and

\footnotetext{
*Corresponding author. Email: xi.jiang@qmul.ac.uk

ORCID(s): 0000-0001-6036-8534 (R.S.M. Freitas); 0000-0001-8035-9651 (F.A. Rochinha);

0000-0001-9901-7942 (D. Mira); 0000-0003-2408-8812 (X. Jiang)
} 
direct numerical simulations (DNS) has been largely extended [3] thanks to the advances in computing power. Nevertheless, the challenge of modeling detailed chemistry is not alleviated.

In general, the chemistry involved in the oxidation process of a reactive mixture can be excessively complex and highly non-linear. A detailed description of a reactive process, hydrocarbon combustion for example, typically involves hundreds or thousands of chemical reactions with hundreds of chemical species [4]. Hence, the use of detailed chemical kinetic models in CFD, including LES or DNS, can pose a significant challenge for the calculation of turbulent reacting flows in complex geometries under engine-relevant conditions, unless techniques for chemistry reduction are used [5-8]. Usually, to reduce the computational cost, reduced reaction mechanisms are employed to describe the kinetics of the combustion process $[4,7,8]$. However, reduced mechanisms can be inadequate under off-design conditions, leading to a detectable inconsistency between models and observations $[9,10]$. Therefore, chemistry modeling represents a major source of uncertainty in computational combustion.

In order to improve the predictability of the kinetics in combustion modeling, a probabilistic perspective can be adopted to describe such uncertainty. Stochastic Bayesian inference in the realm of Uncertainty Quantification (UQ) is a common approach to handle model inadequacy [11-13] and parametric uncertainties. It is considered a powerful framework for combining experimental (or field) data with prior knowledge to develop chemical kinetics models and quantify the associated uncertainties in the model structure [14].

In addition to the errors introduced by computing the reactive chemical process by reduced kinetics, there is a large number of parametric uncertainties in chemical kinetics models, which involve reaction rate constants and thermodynamic parameters, many of which are poorly known [15-17]. They also present strong non-linearities that can intensify small parametric uncertainties, leading to large uncertainties in predictions. Reaction mechanisms capable of describing chemical kinetics must be able to correctly describe the separate chemical evolution of the major species relevant to pollutant formation along with the heat release rate coming from combustion.

The main objective of this study was to investigate the effects of chemical kinetic parameter uncertainties on combustion using a UQ-based methodology. We focus on biogas combustion, a recent area of development. Biogas is a mixture of methane diluted with $\mathrm{CO}_{2}$. The utilization of renewable fuels derived from biomass such as landfills, agricultural wastes, or other sources has received great interest in recent years [18] and is therefore considered here. One of the major problems related to the application of biogas to industrial burners is the low flame stability caused by the dilution of $\mathrm{CO}_{2}$ [19]. There have been some efforts dedicated to understanding the stability of these flames and also its characterization for practical applications [20, 21]. As biogas/air lean premixed flames are likely to play an important role in the future due to its renewable nature, this study is focused on the development of reduced kinetic models for this renewable fuel. In general, the detailed GRIMech3.0 mechanism [22] appears to be appropriate for reliable simulations of biogas combustion containing small quantities of hydrogen [23] and will be used here as the reference mechanism. The evaluation of chemical kinetics on flame parameters conducted in this work corresponds to the application cases of homogeneous combustion during (1) autoignition and (2) flame propagation. These are well-known scenarios from theoretical, experimental, and numerical standpoints. In fact, those conditions are often used in the literature to evaluate the accuracy and reliability of reaction mechanisms [24].

A second objective of the study was to calibrate reduced chemical models of interest with 
respect to a more detailed model, considered as "truth" for the present purpose. We propose two models based on embedding the model discrepancy in Arrhenius kinetic parameters. Finally, we develop a surrogate model of flame propagation using machine learning techniques to make feasible a broader parametric UQ analysis.

This paper is organised as follows. Section 2 introduces the methodology employed to quantify parametric uncertainties. Section 3 presents a model-to-model Bayesian calibration approach to understanding the limits of using reduced model to predict biogas combustion performance. Finally, in section 4, conclusions of the present study are given.

\section{Computational combustion models and reaction mechanisms}

Model discrepancies (also referred to as model errors or structural errors) resulting from employing simplified physics or chemistry tend to be difficult to handle. This is motivated by the need for seeking a balance between easiness of computation and accuracy on complex three-dimensional turbulent reacting flow calculations with LES or DNS. Here, uncertainties are modelled in the context of a probabilistic approach, which means that variables and parameters are assumed to be random variables or processes.

An accurate description of biogas-air combustion requires a detailed chemical mechanism accounting for all relevant elementary reaction steps and intermediates. A widely accepted model for the combustion of biogas is the detailed GRI3.0 mechanism [23, 25], consisting of 325 elementary reactions containing 53 species with $\mathrm{C}_{1}-\mathrm{C}_{2}$ hydrocarbons.

It is well-known that kinetic parameters can have a pronounced effect on the combustion process due to the influence of the oxidation on the resulting physicochemical properties of the reacted gas. Sensitivity analysis of fundamental flame properties to the rate constants can reveal the rate-limiting steps controlling the combustion processes and provide valuable insights into mechanism optimization. In this context, we carry out an analysis of the aforementioned detailed kinetic mechanism from a UQ perspective. The starting point here is the analysis of the effects of kinetics parameters on characteristic flame properties. Furthermore, this study can serve as a basis for further analysis, in an attempt to analyze the impact of the use of simplified mechanisms for renewable fuels searching "best values" in a Bayesian perspective of the kinetic parameters of the most sensitive reactions.

In the following, we start with studying the impact of Arrhenius parameters uncertainties on premixed combustion. That is followed by an analysis of the performance of a simplified mechanism as a proxy of the detailed mechanism GRI3.0. Both models employ the basic scenario of a laminar premixed flame, that consists of 1-D freely propagating laminar flame achieving a steady state. This is the foundation for modelling more complex turbulent combustion. It is often used to determine the main characteristics of specific fuel mixtures such as the flame speed or flame thickness as well as the formation of certain species. The four main variables associated to the steady state that will be considered here are: the flame speed, the temperature of the burnt mixture, the flame thickness and the mass fraction of $\mathrm{CO}$ as an indicator of incomplete combustion process.

\subsection{Model predictive analysis: parametric uncertainties}

The first analysis we perform relies on assuming a stochastic model for the relevant parameters to push forward such uncertainties through the simulations (forward analysis) so as to understand the final impact on quantities of interest $(\mathrm{QoI})$, outputs of the simulation. 
R. S. M. Freitas, F. A. Rochinha, D. Mira \& X. Jiang / Preprint submitted to Chem. Eng. Science

\section{Table 1}

Biogas Composition

\begin{tabular}{ccc}
\hline Constituent (Volume \%) & $\mathrm{CH}_{4}$ & $\mathrm{CO}_{2}$ \\
\hline Baseline & 100 & 0 \\
BG1 & 75 & 25 \\
BG2 & 50 & 50 \\
\hline
\end{tabular}

In practice, kinetic parameters are estimated through indirect, scarce and noisy measurements, so it is natural to think that they bear some level of uncertainty. The aim here is to investigate the impacts caused by small fluctuations in kinetic parameters in practical combustion systems. We assume that the reaction rate $k$ for each elementary step is dictated by the Arrhenius expression:

$$
k(T)=A T^{m} e^{-\frac{E}{R T}},
$$

where $R=8.314 \mathrm{~J} \mathrm{~mol}^{-1} \mathrm{~K}^{-1}$ is the universal gas constant, $T$ is the temperature, $E$ is the activation energy, $A$, a positive value, is the pre-exponential constant and $m$ is a model constant.

Due to the high number of reactions composing the detailed mechanism, a sensitivity analysis was performed to identify those reactions that bear more potential to impact the QoIs by the uncertainties. It helps us on obtaining computational savings along the UQ study. We employed a local sensitivity analysis [26], with respect to the flame speed and flame thickness, employing the normalized derivatives: $\left(k / s_{L} \cdot \partial s_{L} / \partial k\right)$ and $\left(k / \delta_{L} \cdot \partial \delta_{L} / \partial k\right)$. To study such sensitivity on the main flame quantities, three fuel mixtures are considered and presented in Table 1. A baseline fuel containing only methane is included for comparison. Figure 1 shows the flame speed and flame thickness sensitivities associated to the key reactions in the mechanism for the three fuel mixtures. Here, the software Cantera [27] for the simulation of problems involving chemical kinetics, thermodynamics, and transport processes is employed.

The analysis depicted in Fig. 1 reveals that the flame sensitivities are similar for different fuels, where $\mathrm{H}+\mathrm{O}_{2} \Longleftrightarrow \mathrm{O}+\mathrm{OH}$ is the most relevant regarding the uncertainty propagation. Thus, we propose to investigate the impacts caused by small fluctuations of the most sensitive reaction of GRI3.0 mechanism on the flame physicochemical properties. We consider the combustion chemistry as a kinetic problem with a probabilistic structure by assuming the Arrhenius factor $(A)$ and the activation energy $(E)$ of the most relevant reaction as stochastic variables, keeping all other parameters deterministics. Following a probabilistic perspective, we model the stochastic variables as:

$$
\begin{aligned}
& A=\bar{A}\left(1+\sigma_{A} \xi\right), \\
& E=\bar{E}\left(1+\sigma_{E} \xi\right),
\end{aligned}
$$

where $\left(\bar{A}, \sigma_{A}\right)=\left(2.650 \times 10^{16}, 0.05\right) \mathrm{s}^{-1}$ and $\left(\bar{E}, \sigma_{E}\right)=\left(17.041 \times 10^{3}, 0.05\right) \mathrm{cal} / \mathrm{mol}$, while $\xi$ is an independent random variable with uniform distribution $[-1,1]$. Therefore, the domains of variability of the stochastic variables $\mathrm{A}$ and $\mathrm{E}$ are $\left[2.5175 \times 10^{16}, 2.7825 \times 10^{16}\right] \mathrm{s}^{-1}$ and $[16.1889 \times$ 
R. S. M. Freitas, F. A. Rochinha, D. Mira \& X. Jiang / Preprint submitted to Chem. Eng. Science

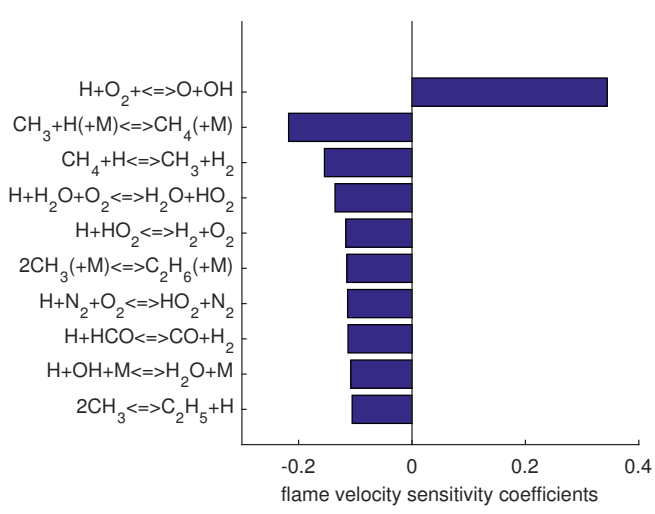

(a) Baseline flame speed sensitivity

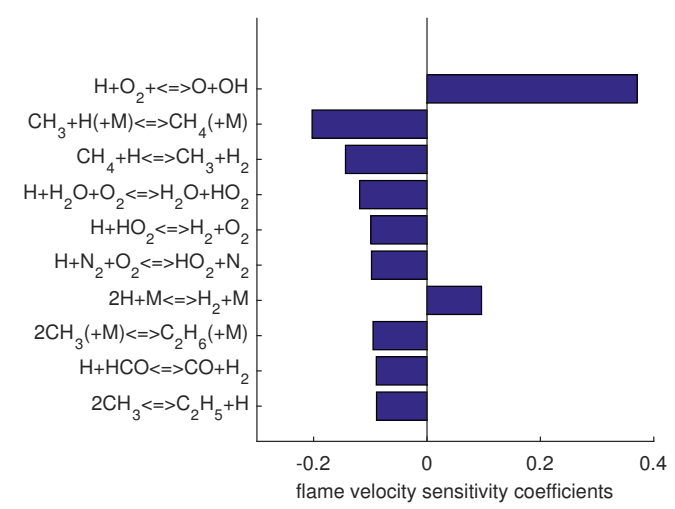

(c) BG1 flame speed sensitivity

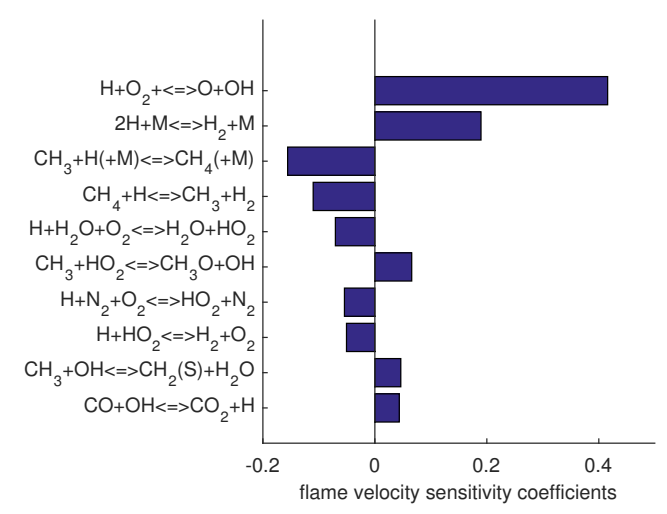

(e) BG2 flame speed sensitivity

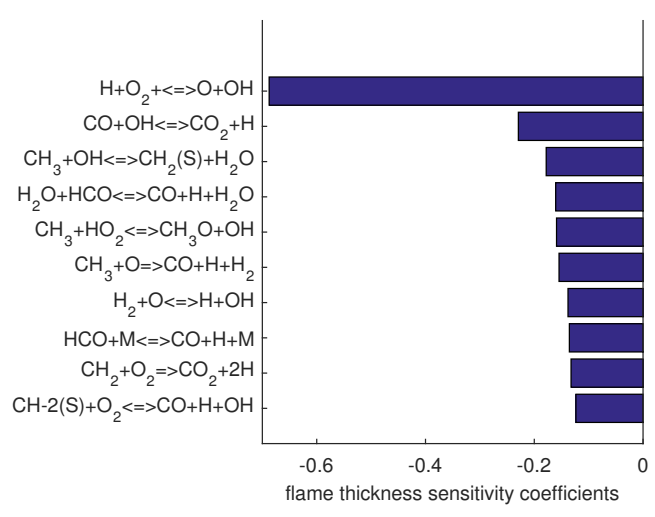

(b) Baseline flame thickness sensitivity



(d) BG1 flame thickness sensitivity

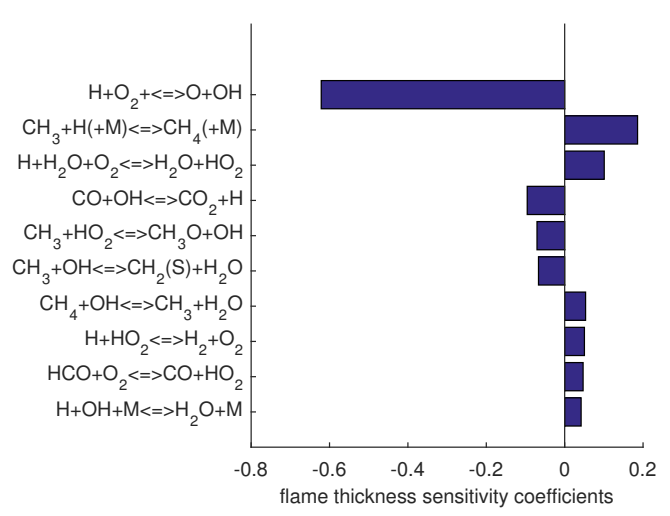

(f) BG2 flame thickness sensitivity

Figure 1: Flame sensitivity analysis of difference fuels at stoichiometric conditions.

$10^{3}, 17.8931 \times 10^{3} \mathrm{c} \mathrm{cal} / \mathrm{mol}$, respectively. The uncertainties are presented in Figure 2 , the results indicate that Arrhenius parameters play a major part in the kinetics, since small fluctuations on Arrhenius parameters cause a high variability on the flame characteristics. Nevertheless, the difference between the three cases is not significant.

Typically, to alleviate the high computational cost of the simulations, reduced reaction mech- 
anisms are employed. The results depicted in Fig. 2 suggest that the use of reduced schemes for methane/air oxidation would be a good starting point to develop optimized schemes for biogas, as the variations in fundamental flame parameters due to composition variations are relatively small and could be captured by the same schemes. In the next section, we present a Bayesian approach to understanding the limits of employing a reduced mechanism for methane/air in this renewable fuel.

\section{Simplified reaction mechanisms: Bayesian analysis}

We now turn the attention towards a different aspect of the model building from a UQ perspective. A critical issue in computational combustion lies on the high computational cost of simulations with detailed chemistry due to the large disparity in time and length scales of the species involved. Typically, simplified models are used as proxies to alleviate such computational burden, and the resulting model is expected to present similar accuracy under certain conditions. Here, we present a model-to-model Bayesian calibration approach to understanding the limits of such low-fidelity models.

For the present purpose, we employ a reduced mechanism based on a 2-steps chemistry that has been widely used in the literature, also referred to as 2S-CM2 [4, 24, 28-30]. This model recovers some of the flame physicochemical properties with high accuracy, but the performance in rich flames is far from satisfactory as the mechanism has not been designed to operate in this regime. Usually, this reduced chemical kinetic mechanism when employed for methane combustion, corrections of the kinetics parameters using empirical adjustments are made to improve the predictions in rich conditions [24, 28, 29]. This reduced scheme will serve as proxy of more detailed kinetic models and is calibrated against the mechanism GRI3.0. The 2-step 2S-CM2 mechanism consists of 6 species with 2 global reactions:

$$
\begin{gathered}
\mathrm{CH}_{4}+\frac{3}{2} \mathrm{O}_{2}=>\mathrm{CO}+2 \mathrm{H}_{2} \mathrm{O} \\
\mathrm{CO}+\frac{1}{2} \mathrm{O}_{2}<=>\mathrm{CO}_{2} .
\end{gathered}
$$

We compare the detailed model outputs with those provided by this reduced mechanism, for the three reference mixtures from Table 1, in Fig 3. This comparison involves the autoignition process of an homogeneous mixture. During autoignition, there is a complex balance of chemical reactions that results in certain delay to achieve equilibrium. This is fundamentally a chemical problem as convection and diffusion are not accounted for. The tested conditions include different equivalence ratios from $\phi \epsilon[0.6,1.4]$ at atmospheric pressure and initial temperatures $T_{0} \in[1000,1300 K]$. For the 2S-CM2 mechanism, we employed typical values for the constant rates [24]. The results are obtained with Cantera.

From these results, we see that the autoignition delay time predicted by the reduced mechanism presents an unsatisfactory agreement leading to a detectable inconsistency in methane and biogas combustion. Therefore, the use of reduced mechanisms needs to be taken with care, as these mechanisms are usually calibrated to match certain properties for a given operation range, but they might lead to significant errors when used outside their application range. A possible approach to 
R. S. M. Freitas, F. A. Rochinha, D. Mira \& X. Jiang / Preprint submitted to Chem. Eng. Science



(a) Baseline flame speed

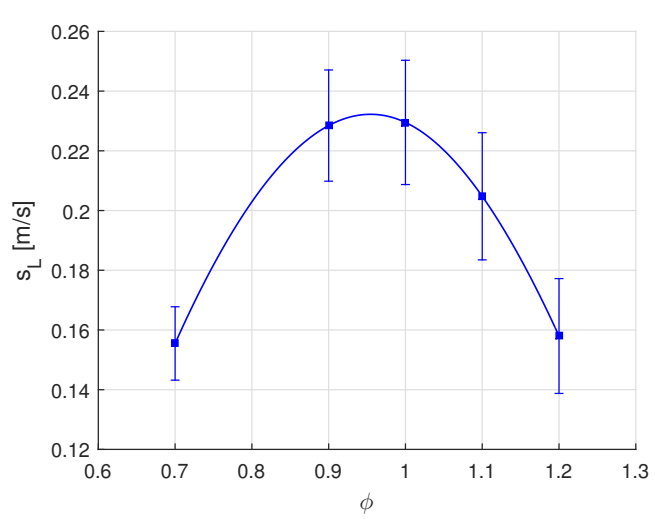

(c) BG1 flame speed

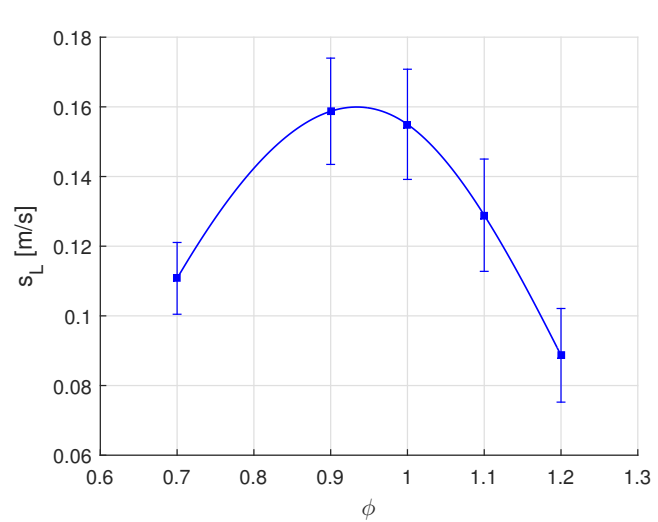

(e) BG2 flame speed

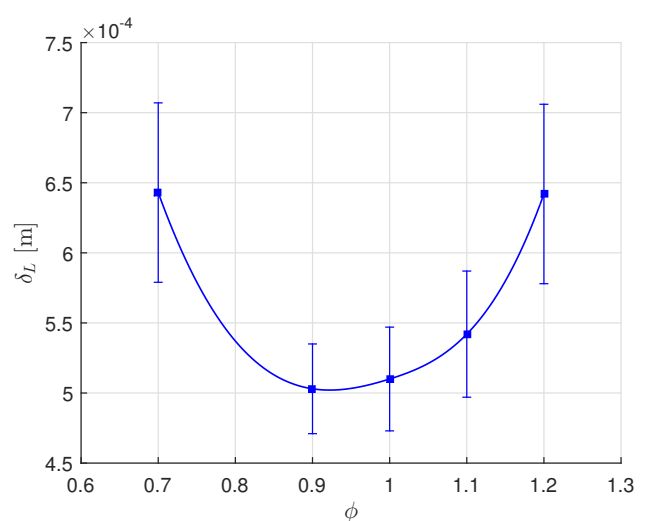

(b) Baseline flame thickness

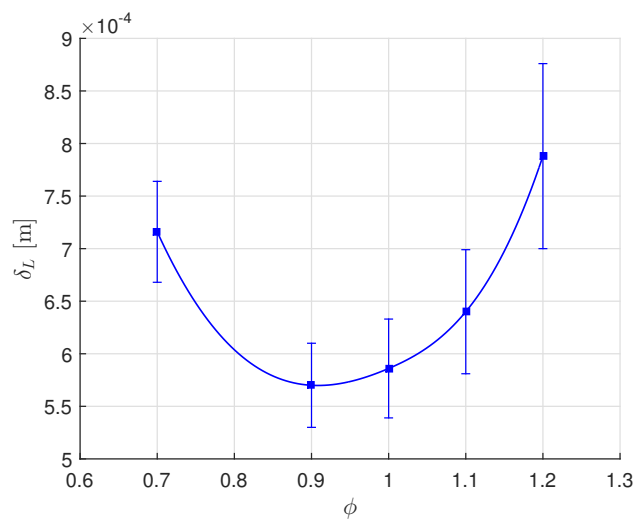

(d) BG1 flame thickness

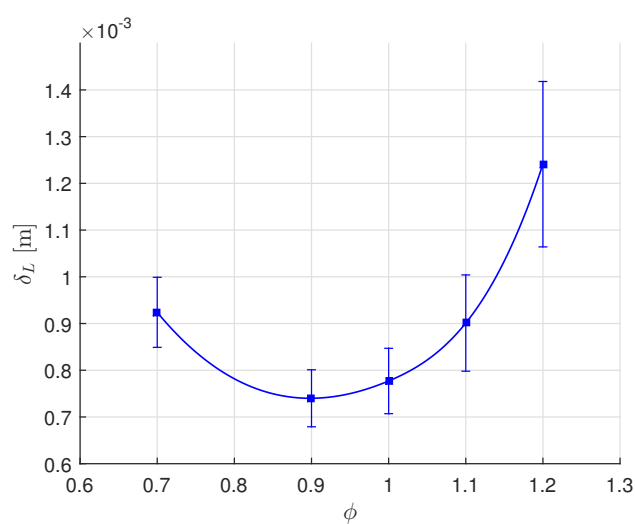

(f) BG2 flame thickness

Figure 2: Influence of fuel composition and Arrhenius parameters on the flame speed and thickness at different equivalent ratios.

address this problem would be to either consider an extended mechanism with the increase in computational cost during CFD simulations, or to provide an optimization of the Arrhenius parameters to account for these effects.

In this context, the use of predictive models based on UQ can be used to extend the applicability 
R. S. M. Freitas, F. A. Rochinha, D. Mira \& X. Jiang / Preprint submitted to Chem. Eng. Science
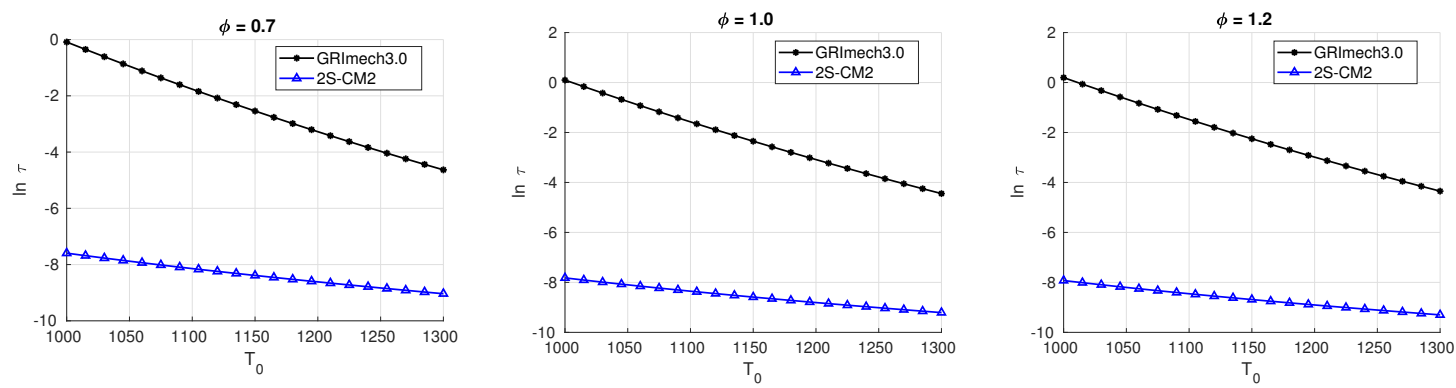

(a) Baseline


(b) BG1
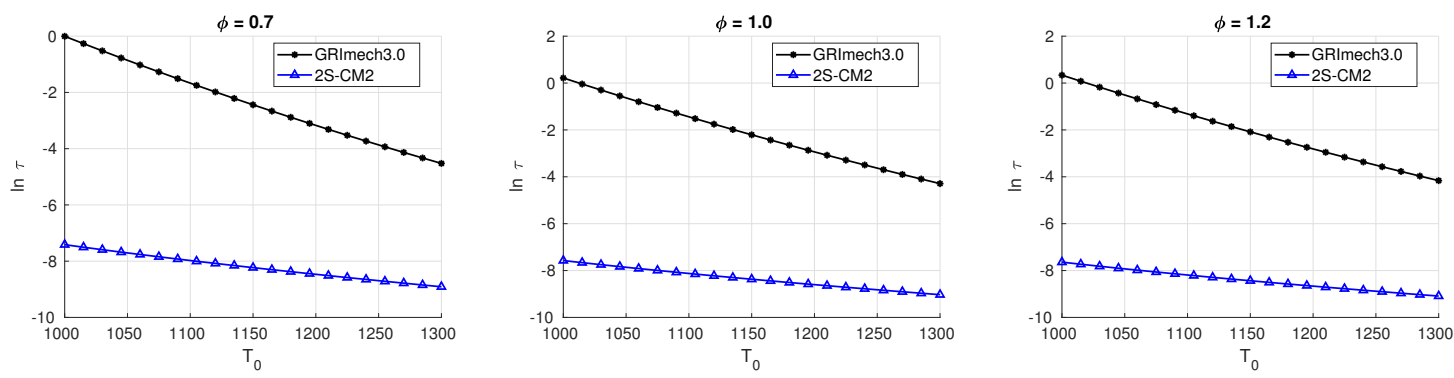

(c) BG2

Figure 3: Auto-ignition delay times for biogas mixtures shown in Table 1.

of reduced schemes to wider operating conditions as an alternative to empirical models. We now start following the preliminary steps of statistical calibration [31,32] assessing if the Arrhenius parameters are suitable for embedding the model discrepancy. For modelling the combustion chemistry of renewable biogas, it is necessary to consider both the detailed model, that capture the key features of biogas chemical reactions [23, 25], and a simplified model. At this point, it is important to highlight that simplified methane models with standard Arrhenius parameters have been tested before for biogas/methane combustion with no success for certain conditions [24, 28, 29, 33]. Here we intend to achieve a better performance through the embedding formulation, at least for laminar flames. We explore this approach in the following sub-sections.

\subsection{Model error characterization}

We employ a Bayesian model calibration approach to assess the performance of the simplified mechanisms. As calibration can be formulated as an inverse problem, Bayesian inference is employed frequently. Within this context, Kennedy and O'Hagan (KO), in their seminal work [34], 
were one of the pioneers to introduce an approach for parameters calibration taking into account model discrepancies. It is built upon an observation equation considering the model discrepancy bias through an additive term, expressed as:

$$
\mathbf{z}=G(\mathbf{s})+\mathbf{e}=f(\mathbf{s}, \boldsymbol{\theta})+\epsilon(\mathbf{s})+\mathbf{e},
$$

where $\mathbf{z}$ is a vector of observable simulation outputs, $s$ is a vector containing input variables that define a scenario $S$ (e.g., boundary and initial conditions, geometry, material properties), $G(\mathbf{s})$ represents the physical truth, and e is the observation noise. The dependence on state variables is omitted to keep the notation simple. Moreover, $f(\mathbf{s}, \boldsymbol{\theta})$ is a short notation for the model, which is a function of the input variable s and parameters to be calibrated $\theta$, and $\epsilon(\mathbf{s})$ denotes the model discrepancy assumed as a Gaussian vector or process. However, the KO approach carries on a number of challenges [31]. First, the imposition of a statistical structure for model discrepancy $\epsilon(\mathbf{s})$ can lead to violation of physical constraints, particularly when considering physics-based models. Another challenge, is that this approach provides a model discrepancy correction to specific cases only. In the case of physical system models intended for predicting numerous quantities of interest, there is no provision for an associated discrepancy correction.

Instead of dealing with these difficulties, we follow a calibration approach [31] that accounts for model discrepancy via probabilistic embedding within the model, in particular the key parameters of the model $f(\mathbf{s}, \boldsymbol{\theta}(\boldsymbol{\alpha}))$. Thus, $\boldsymbol{\theta}$ is a random vector with a PDF depending on hyper-parameters $\boldsymbol{\alpha}$ that one wishes to learn from Bayesian inference. An advantage of this approach, is that since the error is now embedded in the model parameters, it can easily be propagated through the computer model to get the related uncertainty on any quantity of interest impacted by the model parameters.

\subsection{Bayesian calibration}

In order to investigate the impact of model discrepancies due to the use of a reduced mechanism in combustion simulations, we apply a Bayesian calibration approach to handle the model discrepancy. The calibration is based on simulation results produced with the full GRI3.0 detailed mechanism. It is assumed that the data represents the "truth" for a model-to-model calibration. The reference data for calibration is taken from the oxidation process of constant pressure reactors to conduct autoignition delay tests. This choice is motivated by the fact that it is a 0 -D problem and it is chemistry-based, so the effect of convection and diffusion is neglected, which facilitates the calibration of the kinetic parameters. As the reduced mechanism is able to provide satisfactory rates for lean premixed combustion, but not for autoignition, it is also convenient to use it as the starting point. As shown in Fig. 3, the autoignition delays of the reference mixtures have small deviations between them. Thus, the autoignition delay time for the BG1 mixture was chosen, over a range of equivalent ratio $\phi \in[0.6,1.4]$ and a range of initial temperatures $T_{0} \in[1000,1300 \mathrm{~K}]$ varying by 0.1 and $15 \mathrm{~K}$, respectively. Therefore, the reference data for calibration consists of 189 autoignition delay times of biogas/air combustion that will be tested.

\subsubsection{Case 1}

To investigate the limits of the 2S-CM2 mechanism, we employ the probabilistic embedding approach [31]. The irreversible reaction in the $2 \mathrm{~S}-\mathrm{CM} 2$ scheme is the most relevant regarding the flame physicochemical properties. Thus, we take the pre-exponential factor and activation energy of this reaction as the uncertainty (to be identified). Here, we choose the following models for the 
R. S. M. Freitas, F. A. Rochinha, D. Mira \& X. Jiang / Preprint submitted to Chem. Eng. Science activation energy and logarithm of the pre-exponential factor:

$$
\begin{gathered}
E=\theta_{0}, \\
\ln A=\theta_{1},
\end{gathered}
$$

where $\theta_{i}$ are the model parameters that we would like to learn using Bayesian inference.

Given the data $D$, the calibration builds on the following expression derived from the Bayes' formula:

$$
\underbrace{p(\boldsymbol{\alpha} \mid D)}_{\text {posterior }} \propto \underbrace{p(D \mid \boldsymbol{\alpha})}_{\text {likelihood }} \underbrace{p(\boldsymbol{\alpha})}_{\text {prior }}
$$

where $\alpha$ is the vector of parameters describing the inputs $\theta$ as random variables that is to be identified. The prior PDF is chosen to encode accumulated knowledge and will be detailed later on. As the model discrepancy is embedded in the parameters $\boldsymbol{\theta}$, we follow an approach [32] where the model parameters are expanded using a first-order Gauss-Hermite polynomial chaos expansion (PCE) [35]. Thus, a multivariate normal distribution for $\boldsymbol{\theta}$ is adopted:

$$
\begin{gathered}
\theta_{0}=\alpha_{00}+\alpha_{01} \xi_{1}, \\
\theta_{1}=\alpha_{10}+\alpha_{11} \xi_{1}+\alpha_{12} \xi_{2} .
\end{gathered}
$$

Here $\left(\xi_{1}, \xi_{2}\right)$ are i.i.d (independent and identically distributed) standard normal random variables and $\alpha_{i j}$ is the vector of hyper-parameters to be inferred. For the prior, we consider that $\alpha_{i j}$ are i.i.d uniform priors with large boundaries, i.e. noninformative priors, where the starting points of $\alpha_{00}$ and $\alpha_{10}$ are the nominal values of the activation energy and the pre-exponential factor, respectively [24]. Lastly, $\alpha_{1 j} \geqslant 0$, for $1 \leqslant j \leqslant 2$, in order to avoid the sign-flip invariance [31].

The likelihood function connects the parameters and the data through the computer model. In the present work, we assumed an approximate Bayesian computation (ABC) method. The ABC method using a pseudo-likelihood PDF intends to minimise the distance between the computer model mean $\mu_{i}(\boldsymbol{\alpha})$ and the data $D_{i}$ for each data point i, and requires the standard deviation $\sigma_{i}(\boldsymbol{\alpha})$ of the model predictions $f_{i}$ to be consistent with the data spread around the mean model prediction, implying the likelihood form

$$
\mathfrak{L}(D \mid \boldsymbol{\alpha})=\frac{1}{\epsilon \sqrt{2 \pi}} \prod_{i=1}^{N} \exp \left(\frac{\left(\mu_{i}(\boldsymbol{\alpha})-D_{i}\right)^{2}+\left(\sigma_{i}(\boldsymbol{\alpha})-\left|\mu_{i}(\boldsymbol{\alpha})-D_{i}\right|\right)^{2}}{2 \epsilon^{2}}\right)
$$

Here, the tolerance parameter $\epsilon$, which controls the width of type of likelihood is assumed equal to 0.1 [31]. The mean $\mu_{i}(\boldsymbol{\alpha})$ and standard deviation $\sigma_{i}(\boldsymbol{\alpha})$ have to be estimated at each data point $i$ in order to compute the likelihood. We used Chaospy toolbox [36] for performing uncertainty quantification using polynomial chaos expansions, leading to efficient computation of $\mu_{i}(\boldsymbol{\alpha})$ and $\sigma_{i}(\boldsymbol{\alpha})$.

The posterior distribution (6) is complex, which can be characterized by sampling using Markov chain Monte Carlo methods [26, 37, 38]. The algorithm used here to sample from the posterior distribution is Metropolis-Hastings Markov chain Monte Carlo (MCMC) algorithm [39]. A total of 20,000 parameter samples are obtained using MCMC in this study. 
R. S. M. Freitas, F. A. Rochinha, D. Mira \& X. Jiang / Preprint submitted to Chem. Eng. Science
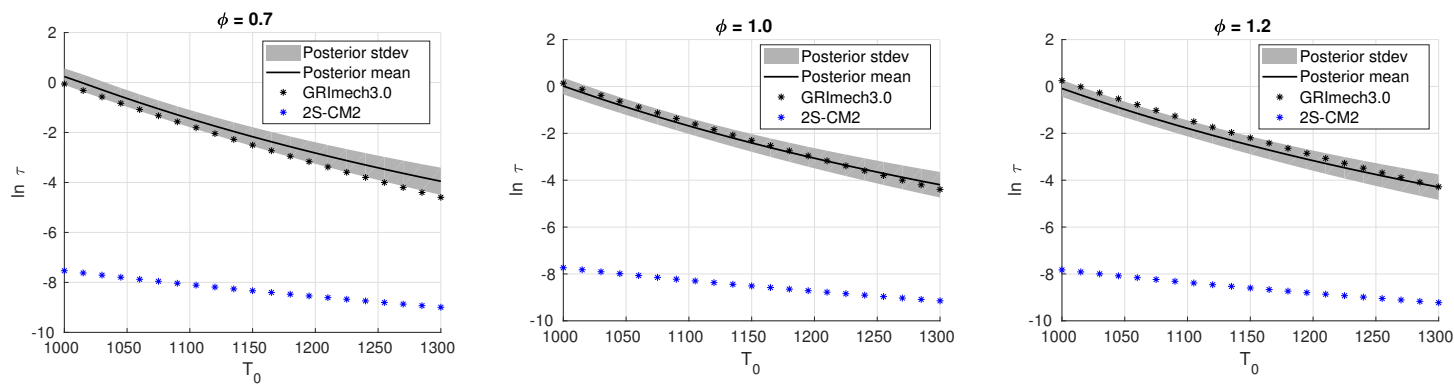

Figure 4: The results of calibration 2S-CM2 model using the GRI3.0 detailed model data, for various values of the equivalent ratio $\phi$.

Using the PDF of vector $\alpha$, we can compute the realizations of $\ln A$ and $E$, i.e. the pushforward of $p(\alpha \mid D)$ through the model. Here, we take 10000 samples of $\alpha$ and, for each of them, 1000 samples of $\xi$ and compute samples of the reaction parameters using the PCE (7). A strong correlation between the Arrhenius parameters can be noted which is consistent with other studies employed Bayesian approach [31, 32, 40].

The associated prediction uncertainties are shown in Fig. 4, where the posterior predictions are illustrated for different equivalent ratios. The figures show that despite the structural deficiencies of the reduced model that prevent it from obtaining the GRI3.0 data in the whole domain, it has predictive error bars that capture the data sufficiently well, given the present calibration procedure.

The calibration process is validated by predicting the autoignition delay time in different scenarios, i.e., the Baseline and BG2 compositions. Figure 5 shows the autoignition delay time of the calibrated mechanism compared to the two-step mechanism and the full GRI3.0. The results indicate a good agreement between the surrogate model and the reference mechanism. The error bars are rather low indicating a high fidelity of the surrogate model for practical applications in the range of temperature and stoichiometry considered. The autoignition delay is a chemical process that depends on the radical pool and requires to account for species associated with the fuel decomposition process like formaldehyde or hydroxide. It is therefore a chemical process difficult to capture by reduced chemical schemes. These results demonstrate the ability of Bayesian inference for model optimizations in chemical kinetics. It is shown how the application range of the reduced model can be extended to predict autoignition without increasing the number of reacting species in the CFD solver and at a reduced computational cost.

The extrapolation of the learning process is now extended to the flame scenario, where we aim to recover the flame properties (flame speed, adiabatic temperature and burned molar fractions of $\mathrm{CO}$ and $\mathrm{CO}_{2}$ ) using the new calibrated model. The predicted values of the flame properties for the freely propagating laminar premixed flames of methane and biogas are shown in Figs. 6 and 7. The results indicate that after calibration for autoignition, the updated 2S-CM2 model can still capture the flame speed and adiabatic flame temperatures in lean and stoichiometric conditions as the original mechanism. The flame speed is slightly overestimated, but the burnt gas temperature and the major combustion products are in good agreement with the original model. The model has been successfully extended to describe flame propagation and autoignition.

However, in rich conditions, the reduced model has structural deficiencies that prevent it from obtaining reliable solutions. Furthermore, it is shown that the variability of model parameters 
R. S. M. Freitas, F. A. Rochinha, D. Mira \& X. Jiang / Preprint submitted to Chem. Eng. Science
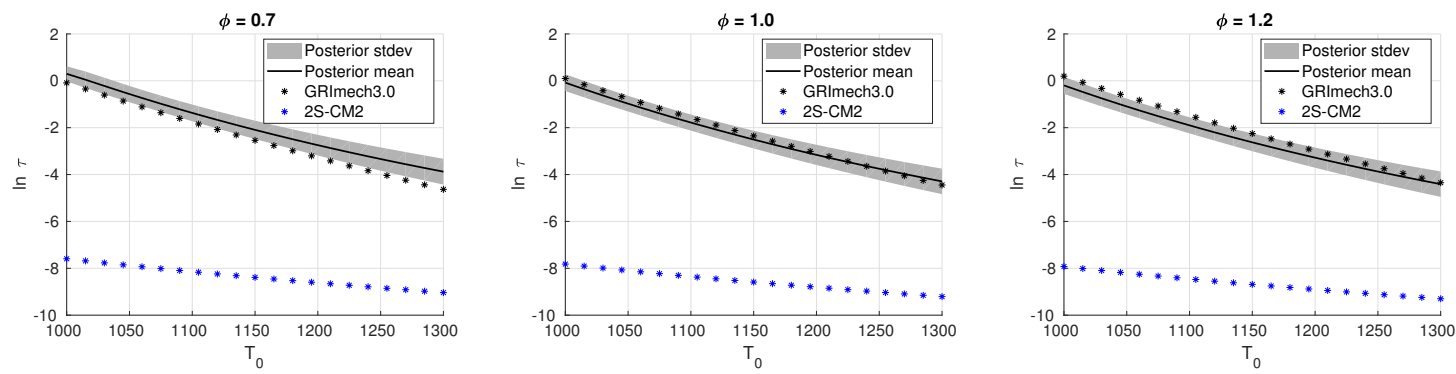

(a) Baseline
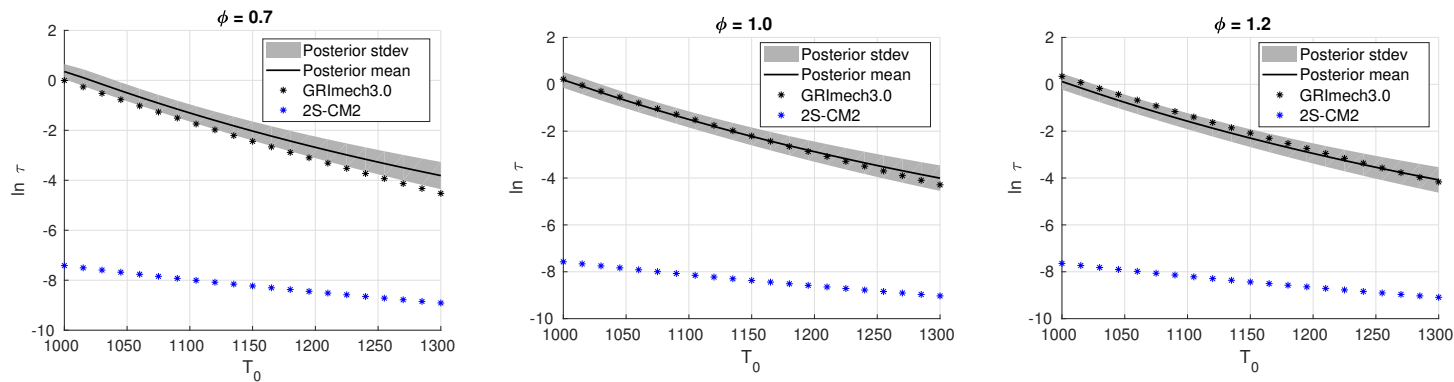

(b) BG2

Figure 5: Autoignition delay time model predictions compared to the GRI3.0: (a) Baseline composition, (b) BG2 composition.

slightly influences the adiabatic flame temperature and only in rich conditions the molar fractions of the combustion products are influenced by the variability of the mechanism. As can be noted in Fig. 7 with the increase of the $\mathrm{CO}_{2}$ in the biogas composition, the molar fraction predictions for rich conditions are unsatisfactory and require further investigation.

\subsubsection{Case 2}

As it has been shown in Case 3.2.1, the enhanced reduced mechanism is not able to reproduce the flame propagation and the species involved in premixed combustion at rich conditions. As proposed by Bibrzycki and Poinsot [24], an empirical correlation based on a pre-exponential factor adjustment (PEA) that redefines the constant rates as a function of the equivalence ratio $\phi$ can be used to recover the correct behaviour in rich conditions. In this section, we apply a Bayesian calibration to this new reduced model as an alternative to PEA in order to extend the applicability range of the reduced scheme to rich conditions and autoignition problems. The corresponding values for activation energy and logarithm of pre-exponential factor follow

$$
\begin{gathered}
E=\theta_{0}=\alpha_{0}+\alpha_{1} \xi_{1}, \\
A=\exp (\ln A) * F, \quad \ln A=\left(\alpha_{10}+\alpha_{11} \xi_{1}+\alpha_{12} \xi_{2}\right),
\end{gathered}
$$

where $F=\beta_{0} \phi^{2}+\beta_{1} \phi+\beta_{2}$ is the pre-exponetial factor adjustment function. We adopted the $\beta_{j}$ values used in the original model [24] for the adjustment function. For the prior, we adopted the same priors of the case 3.2.1 but we limited the $\alpha_{10}$ prior by $5 \%$, i.e., $\alpha_{10}=\bar{\alpha}_{10}(1+0.05 \eta)$, where $\bar{\alpha}_{10}$ is the nominal value of the pre-exponential factor [24] and $\eta$ is an independent random variable with uniform distribution $[-1,1]$. 
R. S. M. Freitas, F. A. Rochinha, D. Mira \& X. Jiang / Preprint submitted to Chem. Eng. Science

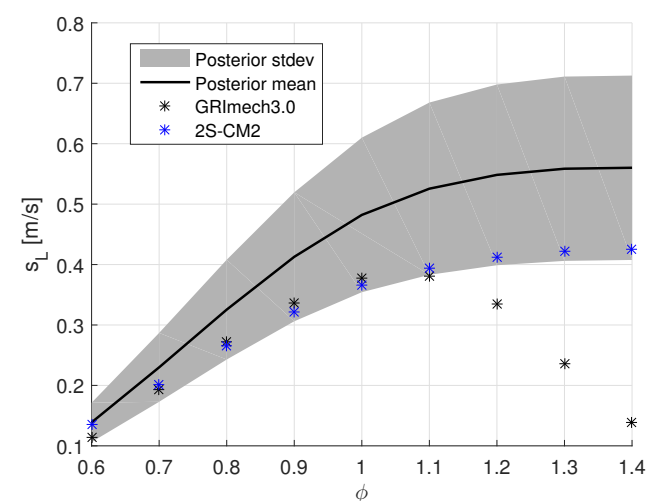

(a) Baseline flame speed

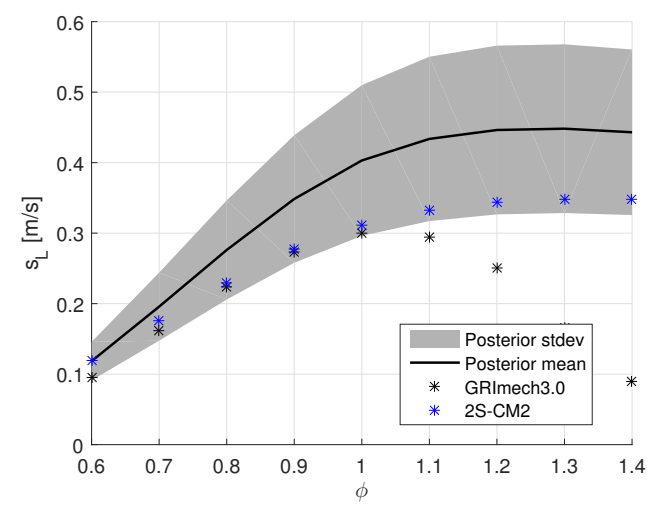

(c) BG1 flame speed

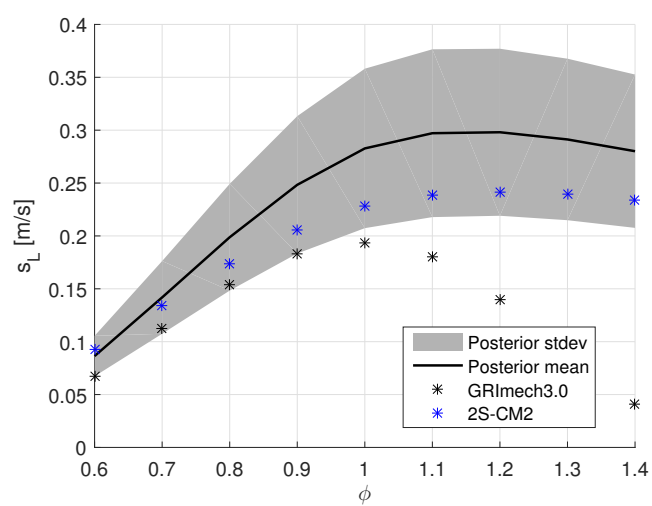

(e) BG2 flame speed

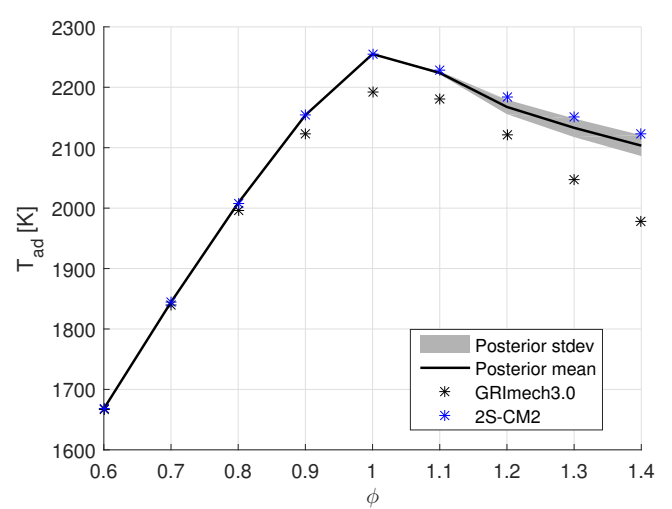

(b) Baseline adiabatic flame temperature

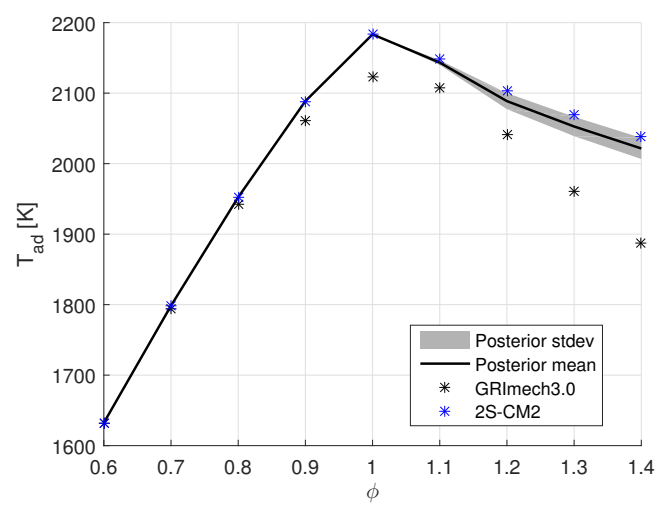

(d) BG1 adiabatic flame temperature

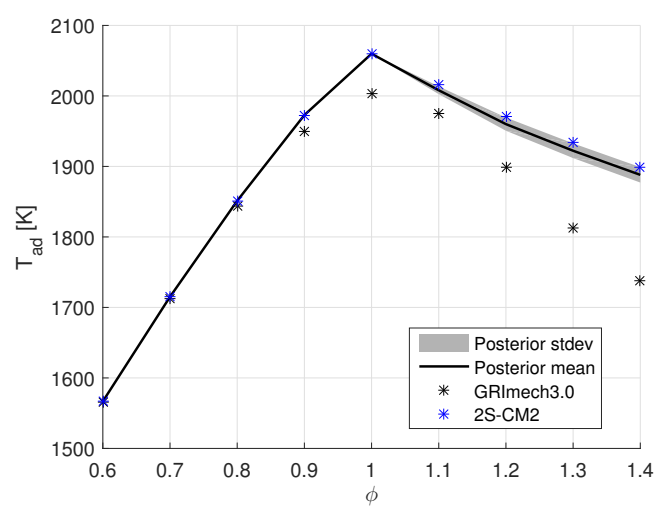

(f) BG2 adiabatic flame temperature

Figure 6: Flame speed and adiabatic temperature predictions for the biogas-air premixed flame of the cases in Table 1 at $p=1$ bar and $T_{0}=300 \mathrm{~K}$.

A total of 20,000 parameter samples are obtained using MCMC, which indicate that there is a strong correlation between the $\ln A$ and $E$, as expected. Despite the structural deficiencies of the reduced mechanism, it has predictive error bars that can reproduce the data from the GRI3.0 data sufficiently well, as shown in Fig. 8. 
R. S. M. Freitas, F. A. Rochinha, D. Mira \& X. Jiang / Preprint submitted to Chem. Eng. Science

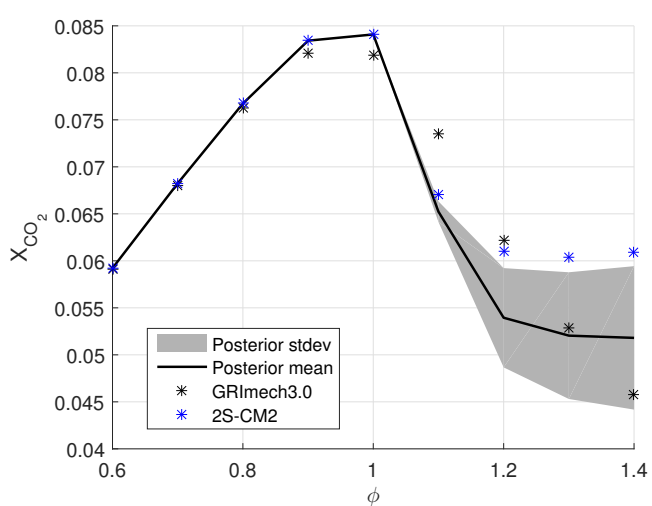

(a) Baseline $\mathrm{CO}_{2}$ burned molar fraction

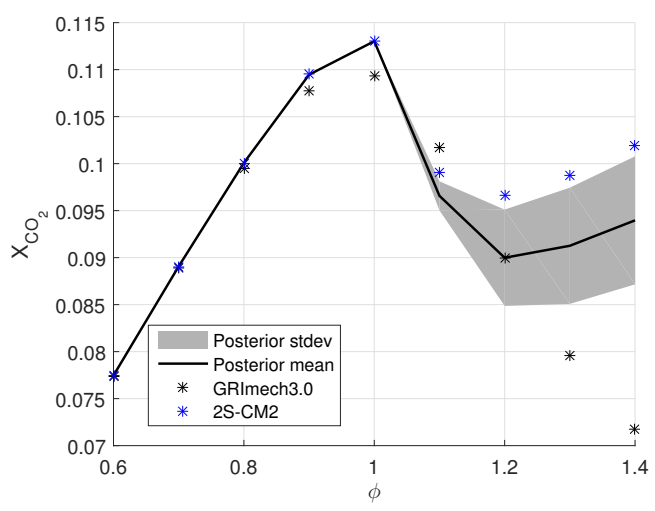

(c) $\mathrm{BG} 1 \mathrm{CO}_{2}$ burned molar fraction

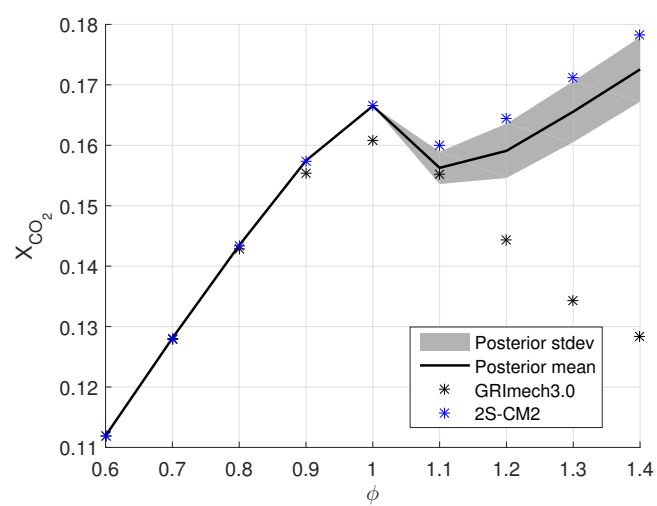

(e) $\mathrm{BG} 2 \mathrm{CO}_{2}$ burned molar fraction

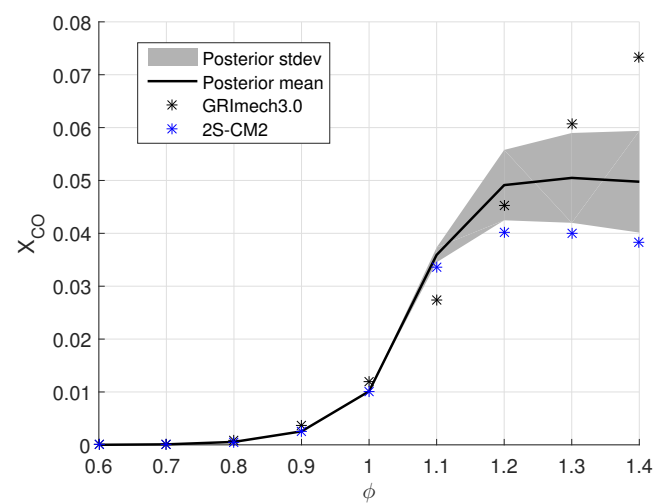

(b) Baseline $\mathrm{CO}$ burned molar fraction

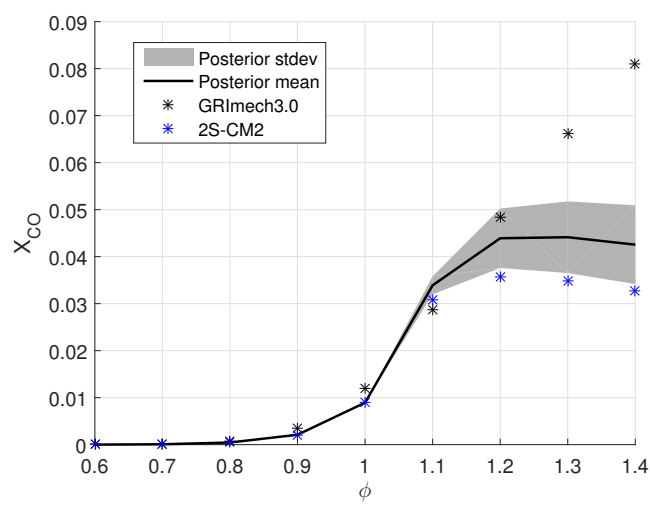

(d) BG1 CO burned molar fraction

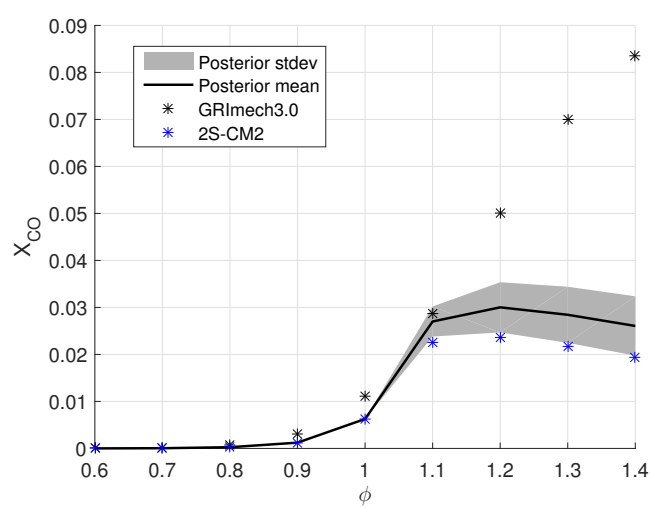

(f) BG2 CO burned molar fraction

Figure 7: Burned molar fractions predictions for the biogas-air premixed flame of the cases in Table 1 at $p=1$ bar and $T_{0}=300 \mathrm{~K}$.

As it was done in Case 3.2.1, the calibration process is validated by predicting the autoignition delay time for the Baseline and BG2 compositions. Figure 9 illustrates the predictions of the calibrated mechanism for these mixtures. It can be noted that the reduced model has predictive error bars that capture the data sufficiently well, given the present calibration procedure. 
R. S. M. Freitas, F. A. Rochinha, D. Mira \& X. Jiang / Preprint submitted to Chem. Eng. Science
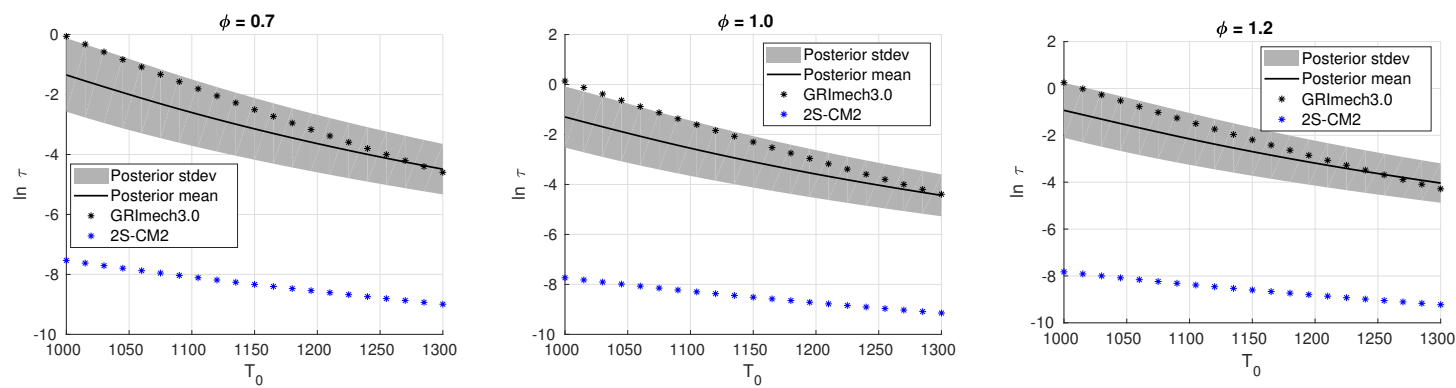

Figure 8: The results of calibration 2S-CM2-PEA model using the GRImech3.0 detailed model data, for various values of the equivalent ratio $\phi$.
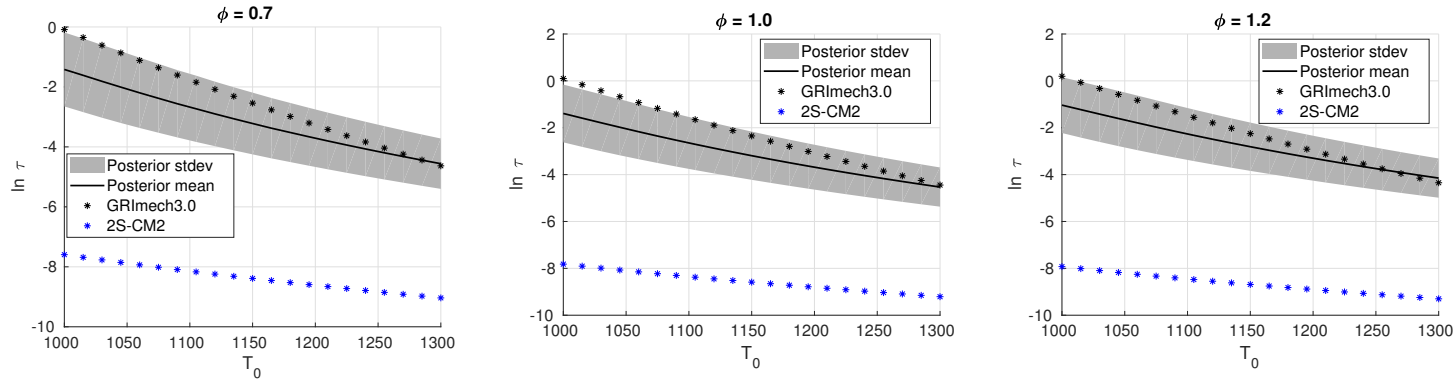

(a) Baseline
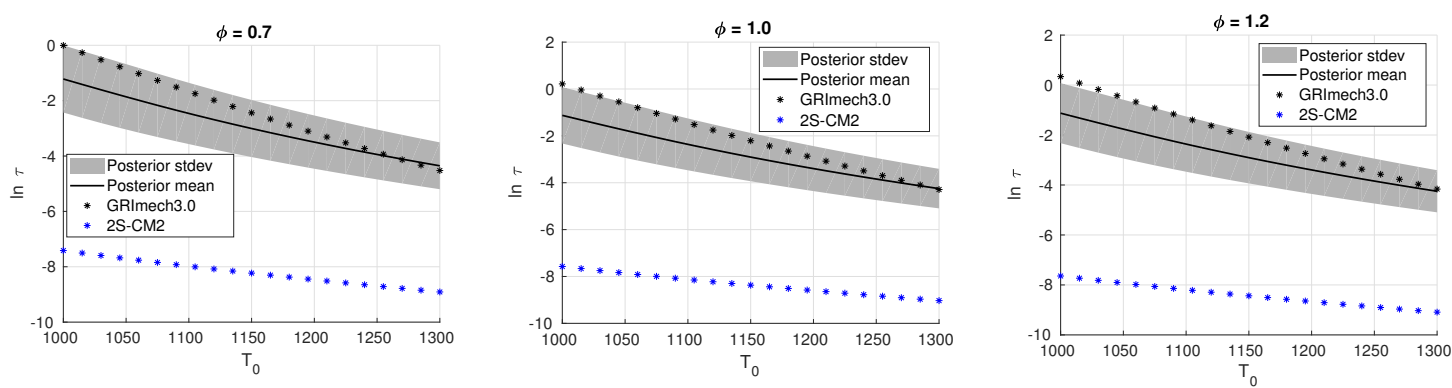

(b) BG2

Figure 9: Autoignition delay time model predictions compared to GRImech3.0: (a) Baseline composition, (b) BG2 composition.

The prediction of properties of the laminar premixed flames for the conditions of the study (see Table 1), is shown in Figs. 10 and 11. The plots show how the fundamental properties of premixed flames are retained after the calibration procedure. However, the calibrated reduced mechanism has structural deficiencies that prevent the model from correctly reproducing the adiabatic flame temperature and combustion products in rich conditions. It can be noted that the variability of model parameters slightly influences the adiabatic temperature and the burned gas compositions. This is further discussed in the next sub-section.

\subsection{Extrapolating to broader operating conditions}

Now, we extend the UQ analysis using a different scenario, and, consequently, we continue with the validation of the calibrated chemical reduced model, leveraging its application to more 
R. S. M. Freitas, F. A. Rochinha, D. Mira \& X. Jiang / Preprint submitted to Chem. Eng. Science

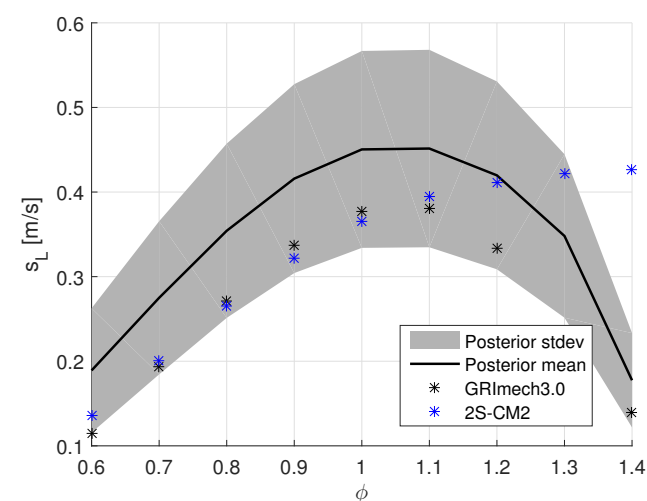

(a) Baseline flame speed



(c) BG1 flame speed

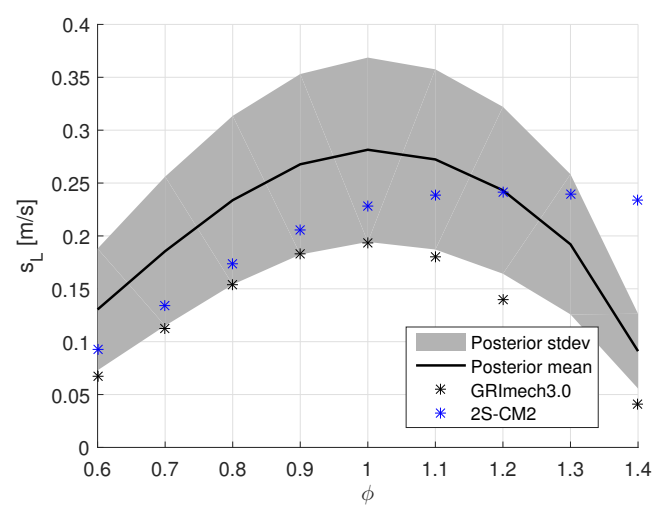

(e) BG2 flame speed

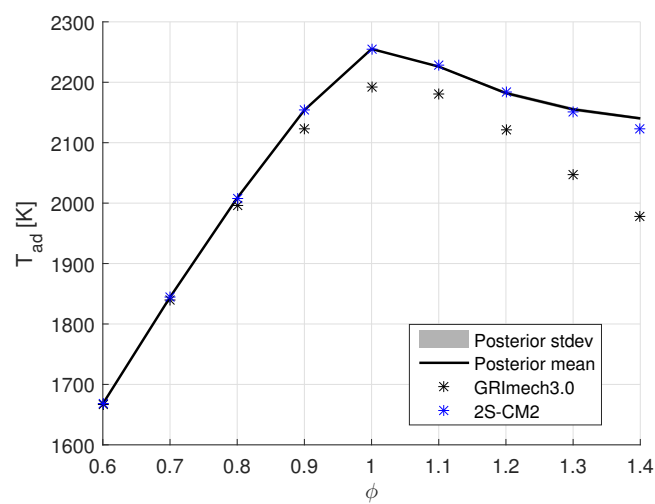

(b) Baseline adiabatic flame temperature

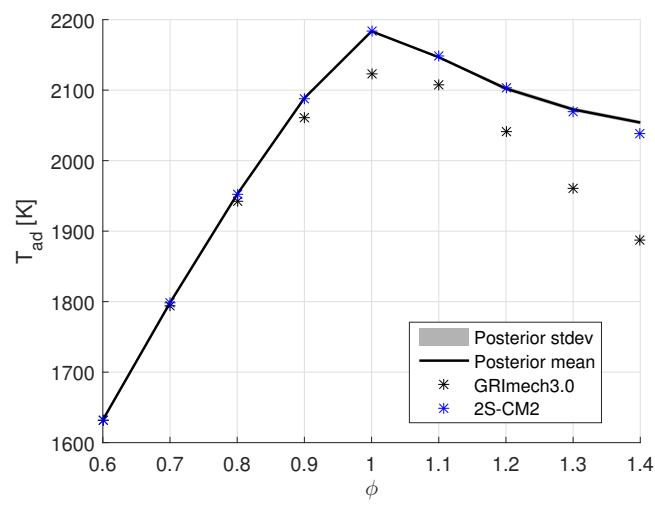

(d) BG1 adiabatic flame temperature



(f) BG2 adiabatic flame temperature

Figure 10: Flame speed and adiabatic temperature predictions for the biogas-air premixed flame of the cases in Table 1 at $p=1$ bar and $T_{0}=300 K$.

complex and challenging simulations. We pursue a traditional route in combustion modeling. After calibrating models in the 0-dimensional homogeneous scenario, we move to a 1-d setting involving steady state laminar flames. Such an approach establishes a hierarchy chain, from low to high fidelity models allowing a gradually consistent way of validation and model improvement. At the 
R. S. M. Freitas, F. A. Rochinha, D. Mira \& X. Jiang / Preprint submitted to Chem. Eng. Science

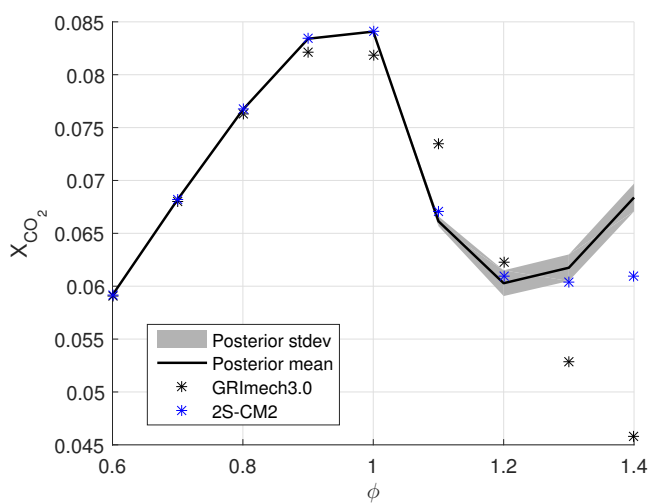

(a) Baseline $\mathrm{CO}_{2}$ burned molar fraction

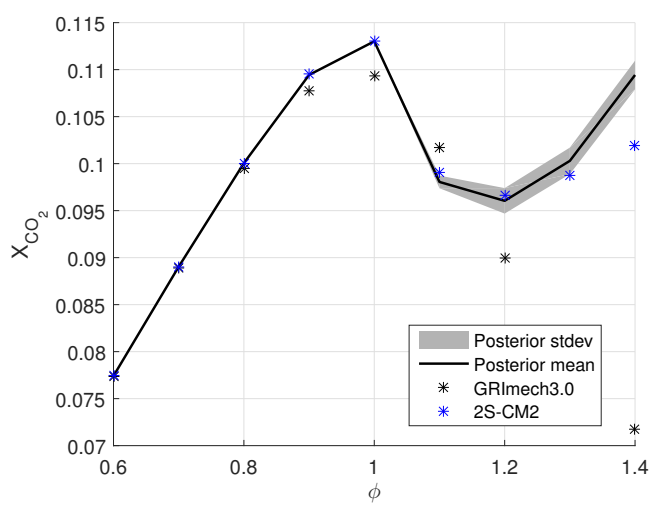

(c) BG1 $\mathrm{CO}_{2}$ burned molar fraction

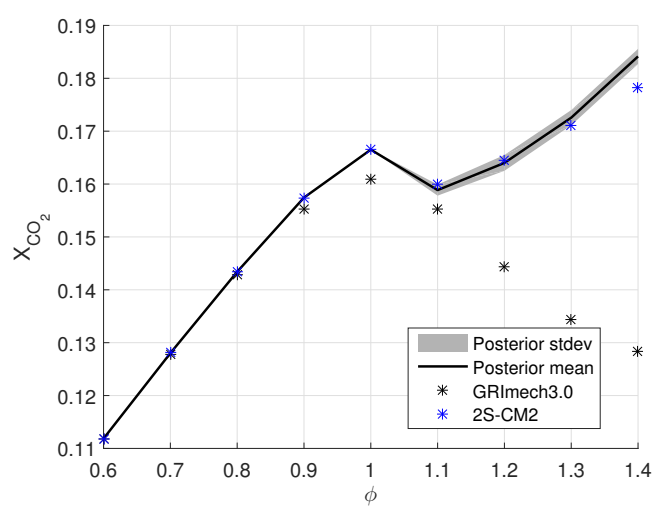

(e) $\mathrm{BG} 2 \mathrm{CO}_{2}$ burned molar fraction

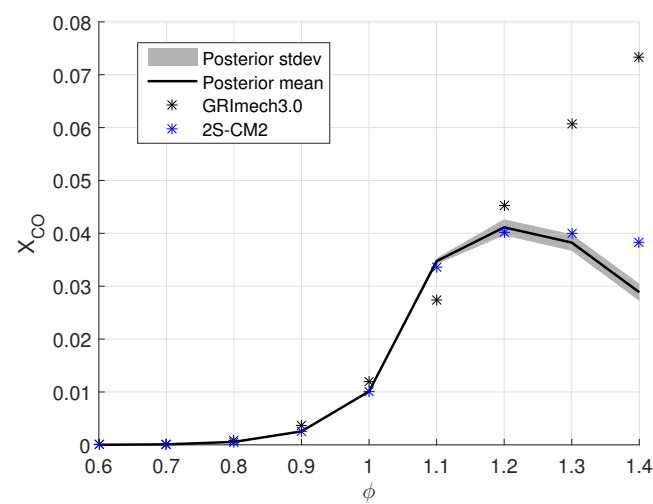

(b) Baseline $\mathrm{CO}$ burned molar fraction

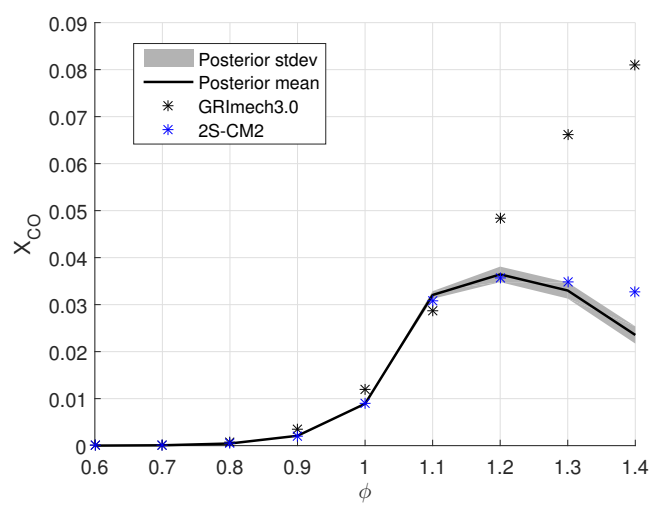

(d) BG1 CO burned molar fraction



(f) BG2 CO burned molar fraction

Figure 11: Burned molar fractions predictions for the biogas-air premixed flame of the cases in Table 1 at $p=1$ bar and $T_{0}=300 K$.

end of the chain, one should have turbulent combustion. The one-dimensional laminar premixed flame is one of the few physics-based computational model that enables direct comparisons between simulation predictions with theory or experiments. It is widely used to validate chemical models and provides foundations to build high-fidelity turbulent combustion approaches, such as flamelet 
R. S. M. Freitas, F. A. Rochinha, D. Mira \& X. Jiang / Preprint submitted to Chem. Eng. Science

based models [41].

More specifically, we aim to investigate, through a UQ perspective, the sensitivity of the flame speed [42], a key quantity of interest in this context, with respect to the operating condition variables: pressure $p$, inlet temperature $T_{0}$, and equivalence ratio of the mixture.This computational analysis might serve different purposes, like optimizing the design of physical experiments under uncertainties [43, 44], to validate models, or to enhance the understanding of the underlying phenomenology [42].

In order to make this analysis feasible, we introduce a machine-learning model [45-50] to alleviate the computational burden of providing flame speed predictions with quantified uncertainty to broader operating conditions. We developed a cheap-to-compute surrogate model to compute the premixed laminar flames using deep densely connected neural networks (Dense Block) [51]. Indeed, Deep Neural Networks (DNN) are becoming a popular tool in producing surrogates in different domains involving physics-based models [45-50], due to their robustness and generalization property.

The machine learning surrogate model is expressed formally in a compact notation as:

$$
\mathbf{y}=f\left(p, T_{0}, \phi, E, \ln A ; \mathbf{w}\right),
$$

with the output $\mathbf{y}$ represents the random flame speed, and $\mathbf{w}$ refers to network parameters to be identified in the network training. The randomness of the flame speed is induced by the calibrated parameters.

The neural network is constructed using the open platform Tensorflow [52]. The dense block presents two design parameters, the number of the layers $L$ and the growth rate $K$. In our surrogate, we consider a dense block with $L=4$ and $K=16$. Training the network means learning the network parameters $\mathbf{w}$ using training data with respect to certain loss function. We adopt a supervised learning strategy, wherein the data set for the training process is provided by simulations of 1-d laminar flame using Cantera software. We use the mean squared error (MSE) [53] for this purpose. To evaluate the ability of the surrogate, we consider the mean relative error (MRE) and coefficient of determination ( $\mathrm{R}^{2}$-score) metrics [54]. The RMSProp optimizer algorithm is used for parameter learning [55], considering a learning rate of $1 \times 10^{-3}$. Finally, it is used 100 epochs in the training process.

In general, combustion systems are operated with leaner mixtures in order to increase the efficiency and reduce the formation of pollutants [56, 57]. Accordingly, the surrogate is constructed bearing that in mind by choosing the surrogate input space of operating conditions: $\phi \in[0.6,1.0]$, $T_{0} \in[300,450] K$, and $p \in[1.0,2.0]$ bar. For the training, we divide this subdomain of the input space with regular partitions defined by $0.2,50 \mathrm{~K}$ and 0.5 bar, respectively. Regarding the calibrated parameters $E$ and $\ln A$, we choose those from the enhanced model of Case 3.2.2. So, we build the training set by randomly selecting 1000 samples of those parameters and combine them with the operating inputs of the regular grid to obtain 36,000 input points, having the corresponding outputs computed using the original flame speed model. Moreover, we use $80 \%$ for training and the remaining $20 \%$ is used for the accuracy assessment of the resulting surrogate model. Such accuracy is verified with the mean relative error that achieves values lower than $0.6 \%$ and the coefficient of determination of 0.997 .

After building the surrogate, we now use the standard Monte Carlo (MC) method to propagate uncertainties arising from the calibration of the Arrhenius parameters into the flame speed. Next, 
we measure the degree of uncertainty of the predictions using the coefficient of variation, defined as the ratio between the standard deviation $\sigma_{y}$ and the mean $\mu_{y}$ of the flame speed

$$
\operatorname{cv}(\mathbf{r})=\frac{\sigma_{\mathbf{y}}(\mathbf{r})}{\mu_{\mathbf{y}}(\mathbf{r})}
$$

where vector $r=\left(p, T_{0}, \phi\right)$ contains the operating condition.

Figure 12 gives an overall picture by displaying a mapping between the operating conditions and the uncertainty on flame speed expressed by the coefficient of variation, after marginalizing out $\ln A$ and $E$ with the use of MC method. We present an explicit quantification of the induced uncertainty resulting from the calibration into the predictions, that helps to understand the limits of the calibrated model when leveraged to a more elaborate setting. More specifically, to make more accessible the visualization of the results, we plot this mapping for six fixed pressures levels, allowing to make explicit the strong dependence of the output uncertainties regarding different levels of such operating input. A critical aspect to highlight in the very beginning is the high values of cv in particular regions of the operating conditions, especially those near the low bound for leaner mixtures. That might be, partially, explained by the burning conditions near flammability limits [58], which bears the potential to amplify the propagated uncertainties. However, it could also be attributed to the calibrated model's use beyond its intrinsic prediction limits. It is not possible to make a definitive judgment without resorting to more data for the calibration process covering such operating conditions, which falls outside this particular study. Also, it is note that variability of flame speed is less pronounced at regions near the stoichiometric condition, while the inlet temperature and pressure level significantly impact the flame speed variability, especially at leaner conditions. All these considerations convey critical information to understand the limitations of the modeling. However, they can also be employed, as mentioned before, in the design of physical experiments, allowing the choice of optimal operating conditions less sensitive to uncertainties contaminating the interpretation of the results.

\section{Conclusion}

In the present study, some aspects and tools are investigated to support the design of computational models dedicated to the combustion of biogas. We employ a UQ probabilistic perspective addressing different sources of uncertainties that might hamper the ability of the model to produce reliable predictions of the combustion process. We analyze such uncertainties sources in two main blocks. The first deals with uncertainties in model inputs (mixture composition) or parameters (Arrhenius relation for the chemical kinetics). We carry out some analysis that confirm the importance of the Arrhenius parameters in the uncertainty in the predictions. That, to a certain extent, inspires the conception of the second block, that is devoted to model discrepancies originated by the use of reduced kinetic models. In that context, we propose two models based on embedding the model discrepancy in such parameters.

The further study not only illustrates a broader scenario regarding the propagation of uncertainties but also demonstrates a key feature of the embedding strategy. Its ability to generate a model that easily adapts to situations significantly different from those used for calibration (or even validation). Moreover, it also serves as a good test for employing a combination of supervised 
R. S. M. Freitas, F. A. Rochinha, D. Mira \& X. Jiang / Preprint submitted to Chem. Eng. Science

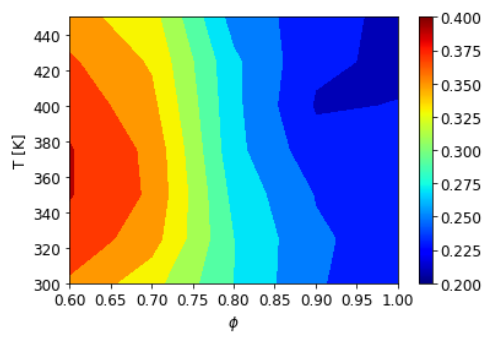

(a) $p=1.0$ bar

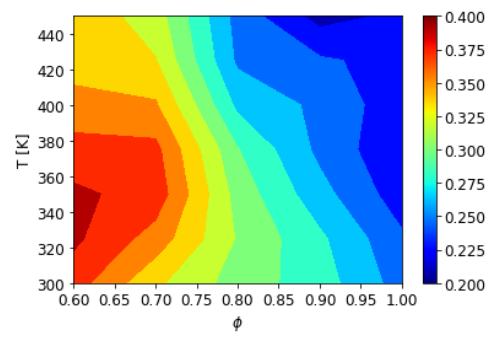

(d) $p=1.6 \mathrm{bar}$

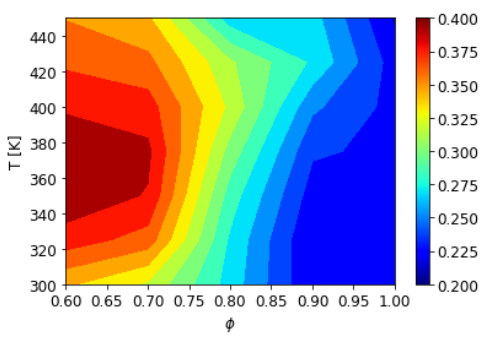

(b) $p=1.2$ bar

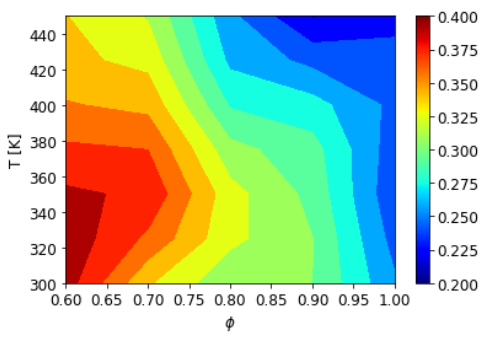

(e) $p=1.8$ bar

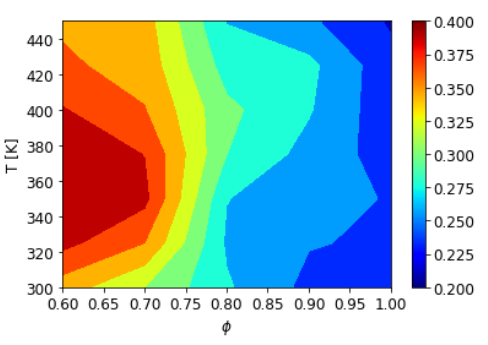

(c) $p=1.4$ bar

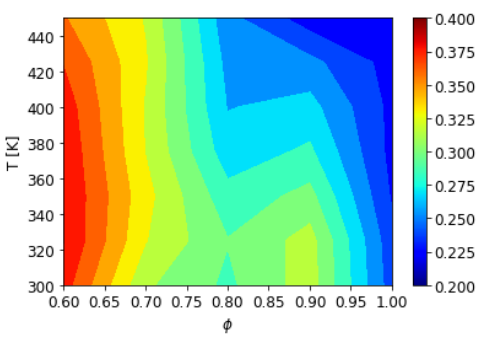

(f) $p=2.0 \mathrm{bar}$

Figure 12: Flame speed variability at different operating conditions.

machine learning techniques with physics-based models to design and construct useful tools to support computational simulation of complex systems.

The study demonstrated the deficiency of the 2-step mechanism and the efficacy of UQ on improving the modelling accuracy. Future studies can be focused on pollutant formation predictions, where more complex chemical kinetic models will be needed.

\section{Acknowledgements}

The research leading to these results had received funding from the European Union's Horizon 2020 Programme (2014-2020) and from Brazilian Ministry of Science, Technology and Innovation through Rede Nacional de Pesquisa (RNP) under the HPC4E Project (www . hpc4e.eu), grant agreement number 689772.

\section{References}

[1] Veynante D. and Vervisch L. Turbulent combustion modeling. Prog. Energy Combust. Sci., 28:193-266, 2002.

[2] Avdic A., Kuenne G., di Mare F., and Janicka J. LES combustion modeling using the eulerian stochastic field method coupled with tabulated chemistry. Combustion and Flame, 175:201-219, 2017.

[3] Chen J. H., Choudhary A., de Supinski B., DeVries M., Hawkes E. R., Klasky S., Liao W. K., Ma K. L., Mellor-Crummey J., Podhorszki N., Sankaran R., Shende S., and Yoo C. S. Terascale direct numerical simulations of turbulent combustion using s3d. Computational Science \& Discovery, 2(1):015001, 2009.

[4] Westbrook C.K. and Dryer F.L. Simplified reaction mechanisms for the oxidation of hydrocarbon fuels in flames. Combustion Science and Technology, 27(10):31-43, 1981.

[5] van Oijen J.A. and de Goey L.P.H. Modelling of premixed laminar flames using flamelet-generated manifolds. Combust. Sci. Technol, 161:113-137, 2000.

[6] Fiorina B., Vicquelin R., Auzillon P., Darabiha N., Gicquel O., and Veynante D. A filtered tabulated chemistry model for les of premixed combustion. Combustion and Flame, 157(3):465 - 475, 2010.

[7] Boivin P., Jimenez C., Sanchez A.L., and Williams F.A. A four step reduced mechanism for syngas combustion. Combust Flame, 158(10):1059-63, 2011.

[8] Williams F.A. Detailed and reduced chemistry for hydrogen autoignition. J. of Loss Prevention in the Process Industries, 21:131-135, 2008. 


\section{R. S. M. Freitas, F. A. Rochinha, D. Mira \& X. Jiang / Preprint submitted to Chem. Eng. Science}

[9] Rebecca E. Morrison, Todd A. Oliver, and Robert D. Moser. Representing model inadequacy: A stochastic operator approach. SIAM/ASA Journal on Uncertainty Quantification, 6:457-496, 2018.

[10] Morrison R. E. Embedded discrepancy operators in reduced models of interacting species. arXiv preprint arXiv:1910.08191, 2019.

[11] Miki K., Prudencio E. E., Cheung S. H., and G. Terejanu. Using bayesian analysis to quantify uncertainties in the $\mathrm{H}+\mathrm{O}_{2}=>\mathrm{OH}+\mathrm{O}$ reaction. Combustion and Flame, 160:861-869, 2013.

[12] Cheung S. H., Miki K., Prudencio E. E., and Simmons C. Uncertainty quantification and robust predictive system analysis for high temperature kinetics of $\mathrm{HCN} / \mathrm{O}_{2} / \mathrm{Ar}$ mixture. Chemical Physics, 475:136-152, 2016.

[13] Miki K., Cheung S. H., Prudencio E. E., and Varghese P. L. Bayesian uncertainty quantification of recent shock tube determinations of the rate coefficient of reaction $\mathrm{H}+\mathrm{O} 2=>\mathrm{OH}+\mathrm{O}$. International Journal of Chemical Kinetics, 475, 2012.

[14] Galagali N. and Marzouk Y. M. Bayesian inference of chemical kinetic models from proposed reactions. Chem. Eng. Science, 123:170-190, 2015.

[15] Mueller M. E., Iaccarino G., and Pitsch H. Chemical kinetic uncertainty quantification for large eddy simulation of turbulent nonpremixed combustion. Proceedings of the Combustion Institute, 2012.

[16] Najm H. N., Debusschere B. J., Marzouk Y. M., Widmer S., and Le Maître O. P. Uncertainty quantification in chemical systems. Int. J. Numer. Meth. Engng, 2009.

[17] Reagan M. T., Najm H. N., Ghanem R. G., and Knio O. M. Uncertainty quantification in reacting-flow simulations through non-intrusive spectral projection. Combustion and Flame, 132:545-555, 2003.

[18] Jiang X., Mira D., and Cluff D. L. The combustion mitigation of methane as a non-co2 greenhouse gas. Progress in Energy and Combustion Science, 66:176-199, 2018.

[19] Dai W., Qin C., Chen Z., Tong C., and Liu P. Experimental studies of flame stability limits of biogas flame. Energy Conversion and Management, 63:157-161, 2012.

[20] Zhen H.S., Leung C.W., and Cheung C.S. A comparison of the heat transfer behaviors of biogas-h2 diffusion and premixed flames. International Journal of Hydrogen Energy, 39:1137-1144, 2014.

[21] Zhen H.S., Leung C.W., Cheung C.S., and Huang Z.H. Characterization of biogas-hydrogen premixed flames using bunsen burner. International Journal of Hydrogen Energy, 39:13292-13299, 2014.

[22] Smith G., Golden D., Frenklach M., Moriarty N., Eiteneer B., Goldenberg M., Bowman C., Hanson R., Song S., Gardiner W., Lissianski V., and Qin Z. Gri mechanism 3.0 website. http://www.me.berkeley.edu/gri-mech/version30/text30.html.

[23] Fischer M. and Jiang X. An investigation of the chemical kinetics of biogas combustion. Fuel, 150:711-720, 2015.

[24] Bibrzycki J. and Poinsot T. Reduced chemical kinetic mechanisms for methane combustion in $\mathrm{O}_{2} / \mathrm{N}_{2}$ and $\mathrm{O}_{2} / \mathrm{CO}_{2}$ atmosphere. Working note ECCOMET WN/CFD/10 17, 2010.

[25] Jiang X. and Fischer M. An assessment of chemical kinetics for bio-syngas combustion. Fuel, 137:293-305, 2014.

[26] Ralph C. Smith. Uncertainty Quantification: Theory, Implementation, and Applications. SIAM in the Computational Science and Engineering Series, CS12, 2014.

[27] David G. Goodwin, Raymond L. Speth, Harry K. Moffat, and Bryan W. Weber. Cantera: An object-oriented software toolkit for chemical kinetics, thermodynamics, and transport processes. https ://www . cantera . org, 2018. Version 2.4.0.

[28] Bibrzycki J. and Poinsot T. Examination of simplified mechanisms of $\mathrm{CH}_{4}$ combustion in $\mathrm{N}_{2} / \mathrm{O}_{2}$ and $\mathrm{CO}_{2} / \mathrm{O}_{2}$ atmosphere using mathematical modeling. Archivum Combustionis, 31:255-262, 2011.

[29] Bibrzycki J., Poinsot T., and Zajdel A. Investigation of laminar flame speed of $\mathrm{CH}_{4} / \mathrm{N}_{2} / \mathrm{O}_{2}$ and $\mathrm{CH}_{4} / \mathrm{CO}_{2} / \mathrm{O}_{2}$ mixtures using reduced chemical kinetic mechanisms. Archivum Combustionis, 30:287-296, 2010.

[30] Boudier G. Methane/air flame with 2-step chemistry: $2 \mathrm{~S}-\mathrm{CH}_{4}-\mathrm{CM}$ 2. Tech. report, CERFACS, 2007.

[31] Sargsyan K., Najm H. N., and Ghanem R. On the statistical calibration of physical models. International Journal of Chemical Kinetics, 47:246-276, 2015.

[32] Hakim L., Lacaze G., Khalil M., Najm H. N., Sargsyan K., and Oefelein J. C. Probabilistic parameter estimation in a 2-step chemical kinetics model for n-dodecane jet autoignition. Combustion Theory and Modelling, 47:246-276, 2018.

[33] Luigi Acampora, Francesco Marra, and Emanuele Martelli. Comparison of different CH4-air combustion mechanisms in a perfectly stirred reactor with oscillating residence times close to extinction. Combustion Science and Technology, 188:707-718, 052016.

[34] Kennedy M. C. and O'Hagan A. Bayesian calibration of computer models. Journal of the Royal Statistical Society: Series B (Statistical Methodology), 2001.

[35] Wiener N. The homogeneous chaos. Amer. J. Math., pages 897-936, 1938.

[36] Feinberg J. and Langtangen H. P. Chaospy: An open source tool for designing methods of uncertainty quantification. J. of Computational Science, 11:46-57, 2015.

[37] W. R. Gilk, Richardson S., and Spiegelhalter D. J. Markov chain monte carlo in practice. Chapman \& Hall, 1996.

[38] Cowles M. K. and Carlin B. P. Markov chain monte carlo convergence diagnostics: A comparative review. American Statistical Association, 91:883-904, 1996.

[39] Kaipio J. and Somersalo E. Statistical and Computational Inverse Problems. Applied Mathematical Sciences, volume 160. Springer-Verlag, 2004.

[40] Hakim L., Lacaze G., Khalil M., Najm H. N., and Oefelein J. C. Modeling auto-ignition transients in reacting diesel jets. Proceedings of the ASME 2015 Internal Combustion Engine Division Fall Technical Conference, 2015.

[41] Poinsot T. and Veynante D. Theorical and numerical combustion. R.T. Edwards, Inc., second edition, 2005.

[42] Shuang Li, Bin Yang b, and Fei Qi. Accelerate global sensitivity analysis using artificial neural network algorithm: Case studies for combustion kinetic model. Combustion and Flame, 168:53-64, 2016.

[43] Chen Z. On the accuracy of laminar flame speeds measured from outwardly propagating spherical flames: Methane/air at normal temperature and pressure. Combustion and Flame, 162:2442-2453, 2015. 


\section{R. S. M. Freitas, F. A. Rochinha, D. Mira \& X. Jiang / Preprint submitted to Chem. Eng. Science}

[44] Christodoulos Xiouris, Tailai Ye, Jagannath Jayachandran, and Fokion N. Egolfopoulos. Laminar flame speeds under engine-relevant conditions: Uncertainty quantification and minimization in spherically expanding flame experiments. Combustion and Flame, 163:270-283, 2016.

[45] Tripathy R. and Bilionis I. Deep uq: Learning deep neural network surrogate models for high dimensional uncertainty quantification. Journal of Computational Physics, 375:565-588, 2018.

[46] Karumuri S., Tripathy R., Bilionis I., and Panchal J. Simulator-free solution of high-dimensional stochastic elliptic partial differential equations using deep neural networks. Journal of Computational Physics, 404, 2020.

[47] Zhu Y. and Zabaras N. Bayesian deep convolutional encoder-decoder networks for surrogate modeling and uncertainty quantification. Journal of Computational Physics, 366:415-447, 2018.

[48] Mo S., Zhu Y., Zabaras N., Shi X., and Wu J. Deep convolutional encoder-decoder networks for uncertainty quantification of dynamic multiphase flow in heterogeneous media. Water Resources Research, 55:703-728, 2019.

[49] Geneva N. and Zabaras N. Quantifying model form uncertainty in reynolds-averaged turbulence models with bayesian deep neural networks. Journal of Computational Physics, 383:125-147, 2019.

[50] Zhu Y., Zabaras N., Koutsourelakis P.S., and Perdikaris P. Physics-constrained deep learning for high-dimensional surrogate modeling and uncertainty quantification without labeled data. Journal of Computational Physics, 394:56-81, 2019.

[51] Huang G., Liu Z., van der Maaten L., and Weinberger K. Q. Densely connected convolutional networks. Proceedings of the IEEE Conference on Computer Vision and Pattern Recognition, 2017.

[52] Martín Abadi, Ashish Agarwal, Paul Barham, Eugene Brevdo, Zhifeng Chen, Craig Citro, Greg S. Corrado, Andy Davis, Jeffrey Dean, Matthieu Devin, Sanjay Ghemawat, Ian Goodfellow, Andrew Harp, Geoffrey Irving, Michael Isard, Yangqing Jia, Rafal Jozefowicz, Lukasz Kaiser, Manjunath Kudlur, Josh Levenberg, Dandelion Mané, Rajat Monga, Sherry Moore, Derek Murray, Chris Olah, Mike Schuster, Jonathon Shlens, Benoit Steiner, Ilya Sutskever, Kunal Talwar, Paul Tucker, Vincent Vanhoucke, Vijay Vasudevan, Fernanda Viégas, Oriol Vinyals, Pete Warden, Martin Wattenberg, Martin Wicke, Yuan Yu, and Xiaoqiang Zheng. TensorFlow: Large-scale machine learning on heterogeneous systems, 2015. Software available from tensorflow.org.

[53] Chollet F. Deep Learning with Python. Manning Publications Company, 2017.

[54] Sanford Weisberg. Applied Linear Regression. John Wiley \& Sons, Inc., 2005.

[55] Gandhi R. A look at gradient descent and rmsprop optimizers: A brief explanation. https://towardsdatascience.com/a-look-at-gradient-des 2018.

[56] Gövert S., Mira D., Kok J. B. W., Vázquez M., and Houzeaux G. The effect of partial premixing and heat loss on the reacting flow field prediction of a swirl stabilized gas turbine model combustor. Flow, Turbulence and Combustion, 100:503-534, 2018.

[57] Li S., Li S.H., Mira D., Zhu M., and Jiang X. Investigation of dilution effects on partially premixed swirling syngas flames using a LES-LEM approach. Journal of the Energy Institute, 91:902-915, 2018.

[58] Chang-Eon Lee and Cheol-Hong Hwang. An experimental study on the flame stability of lfg and lfg-mixed fuels. Fuel, 86(5-6):649-655, 2007. 